\title{
Performance Management in Broadband Satellite Multimedia Networks
}

\author{
Matteo Berioli \\ DLR, Germany \\ matteo.berioli@dlr.de
}

\author{
Robert J. Mort \\ Systek Consulting Ltd., UK \\ robert.mort@etsi.org
}

\author{
Haitham Cruickshank, \\ University of Surrey. U.K. \\ H.Cruickshank@surrey.ac.uk
}

\begin{abstract}
The approach currently being taken by ETSI (BSM) to standardisation for Performance Management (and in particular monitoring) is described. This paper describes parameters, instruments and methods that can be used to manage a BSM sub-network. The final objective is to arrive at a consensus for a standard on this subject.
\end{abstract}

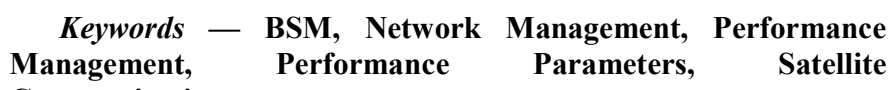
Communications

\section{INTRODUCTION}

Network Performance Management is an important aspect of overall Network Management. The architecture and requirements for Network Management of Broadband Satellite Multimedia (BSM) systems has been defined in [1]

The FCAPS model defined by the ITU is used as a basis for the network management functions, representing:

- Faults

- Configuration

- Accounting

- Performance

- Security.

This paper deals with Performance aspects.

Network performance management strategy is shifting from relatively simple availability of measurements to those based on detailed Quality-of-Service (QoS) performance. This is because network element availability is generally high and stable, with hardware or software infrastructure failures occurring infrequently. At the same time increasing variety of traffic (e.g. voice, video and data), and multi-tiered applications have led to an increase in the volume and complexity of network traffic.

Hence network management is moving from the limited perspective of "device-aware" network management to focus on application delivery - an "application-aware" perspective that is both more comprehensive and more cost-effective. Being application-aware means understanding the content flowing over the network. Once armed with that understanding, traffic flows can be prioritised and devices configured based on the value and priority of the important content.
Whereas this is true for satellite as well as terrestrial networks, normally the satellite link represents, from the performance management point of view, the weak link in the end-to-end chain; thus one of the key objectives of Performance Management in satellite networks is ultimately to ensure that Service Level Agreements between the satellite service provider (Satellite Network Operator [SNO], Network Access Provider [NAP] etc.) and other service providers are met. Hence the aspects of Performance Management which are of interest to different actors in the network need to be taken into account here.

Generally, network performance is determined from network traffic measurements in two ways:

- Active measurements are performed by injecting traffic with known properties into the network;

- Passive measurements consist of monitoring the existing traffic flow(s) at one or more points.

In terms of performance management, QoS measurements lie logically above network traffic measurements, and relate to the performance of networking applications:

- Objective QoS relates to something concrete and quantitative (e.g. Packet loss, Delay, Jitter, Connection break length...);

- Subjective QoS corresponds to the service quality from the user perspective (Mean Opinion Score (MOS) tests are often used); subjective QoS can be estimated within certain limits from the basis of objective QoS (e.g., PESQ algorithm).

The performance parameters to be considered in BSM networks are those described in the ETSI BSM document ETSI TR 102673 [2]. The objectives of Performance Management can be divided into the following categories, or function set groups (in ITU terminology):

- Performance Quality Assurance,

- Performance Monitoring,

- Performance Management Control,

- Performance Analysis.

Performance Quality Assurance

Performance Quality Assurance supports decision processes that establish, according to current state-of-the-art, SLAs, and customer needs, the quality measures that are appropriate for a 
correct performance management. It concerns setting the alert thresholds, selecting the types of test and the frequency with which to perform tests, etc. It is totally up to the satellite network operator how to deal with these decisions, which are therefore considered out of the scope of the paper.

\section{Performance Monitoring}

Performance Monitoring (PM) involves the continuous collection of data from the BSM network elements. The basic function of PM is to track system, network or service activities in order to gather the appropriate data for determining performance of the BSM network. The operation is designed to constantly measure the overall quality of the network connections; using monitored parameters in order to timely detect service degradation. It may also be designed to detect characteristic patterns of impairment before service quality has dropped below an acceptable level. These are key operations should be considered when defining a set of standard M-plane functions in BSM networks.

\section{Performance Management Control}

Performance Management Control supports the transfer of information to control the operation of the network for a correct performance management. For example it deals with the management of measurement schedules, alert thresholds, and other attributes for monitoring and for test traffic. These are also key operations to be considered when defining a set of standard M-plane functions in BSM networks.

\section{$\underline{\text { Performance Analysis }}$}

Performance analysis deals with the measurement data which may require additional processing and analysis in order to evaluate the performance level of the entity. This kind of analysis which depends on, and it is in fact derived from, the type of measurement performed.

\section{PERFORMANCE PARAMETER MEASUREMENT}

Regarding measurement of performance parameters in satellite networks, although the satellite IP performance parameters can represent a classical metrics for IP networks, the way to measure these parameters is not straightforward in general, and it may not be trivial at all in satellite networks, mainly for the following reasons:

- Chattiness: exchange of additional data over the satellite has always to be done with care;

- Scalability: a central server collecting data from a number of observation points in a BSM network may have the need to distinguish some thousands of metering processes;

- Timestamp synchronization: for STs, which are very far from each other and which can only be reached through the satellite link, it may be not easy to synchronize the timestamps with the resolution requested in traditional network management (e.g. at least the one of the sysUpTime in SNMP, which is one hundredth of a second).

So concerning the practical estimation of the performance parameters, it should be noted that there are protocols which allow exchanging measurements in standard formats, and thus giving the possibility to collect data from heterogeneous devices from different vendors. These issues will also be addressed in the full paper.

Figure 1 shows the terminology to be used for BSM performance parameters. The BSM performance parameters always refer to BSM elements (e.g. Satellite Terminals, STs) or to portions of the BSM network. Measurement Points (MPs) for these performance parameters (the BSM MPs) are in general associated to unique unicast IP addresses: A BSM MP can be defined at every layer-2 service access point in an IP host belonging to a BSM network (e.g. an ST, a hub, or even the satellite if it implements on-board routing). As a consequence BSM MPs coincide with Satellite Independent Service Access Points (SI-SAPs), in the hosts where the SISAP is implemented. This means that standard IP properties can be observed at BSM MPs, as well as SI-SAP specific properties, which are more related to SD events.

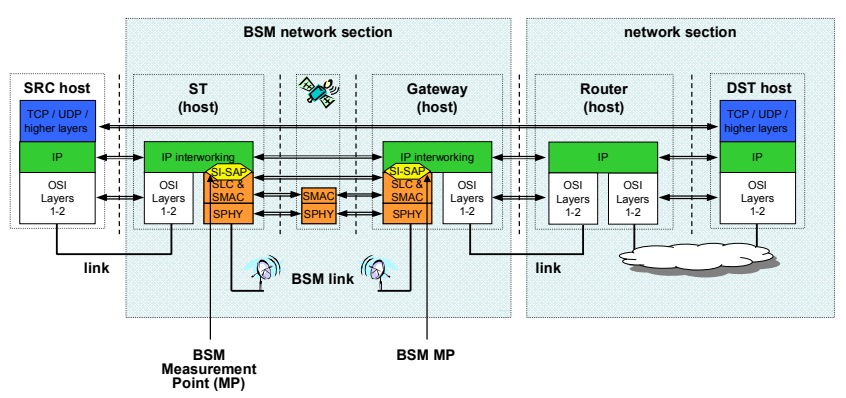

Figure 1: Terminology for BSM performance parameters.

The BSM parameters can be classified in two groups:

- BSM SI-SAP parameters: They are specific to the BSM, i.e. will be available at the SI-SAP and relate to lower protocol layers; they do not refer to IP-level performance, but to more specific properties of one terminal, or of one service class being provided on one particular link.

- BSM SLA parameters: They are the typical IP-layer parameters, defined in a way similar to ITU, but with respect to a specific BSM section or a BSM link between two given devices (e.g. between two STs).

The above performance parameters will be examined elaborated in detailed in this paper.

\section{INFORMATION MODEL FOR PERFORMANCE MANAGEMENT}

BSM Performance Management will focus on IP-layer measurements.

Considering the BSM management functional architecture shown in Figure 2, it is assumed that two types of database $(\mathrm{D} / \mathrm{B}), \mathrm{D} / \mathrm{B}_{1}$ and $\mathrm{D} / \mathrm{B}_{2}$ will be normally used in $\mathrm{BSM}$ networks. $\mathrm{D} / \mathrm{B}_{1}$ and $\mathrm{D} / \mathrm{B}_{2}$ are understood to be combinations of information models (e.g. MIBs), both standard and proprietary ones, as will be explained in the following. 
The internal format of $\mathrm{D} / \mathrm{B}_{1}$ and $\mathrm{D} / \mathrm{B}_{2}$ is not relevant for the scope of this document. The present specification is only concerned with the type of information transferred between management functions to populate these databases, or in other words the relationships (associations) between these entities and the roles identified in a BSM network.

$\mathrm{D} / \mathrm{B}_{1}$ contains a newly specified BSM-specific MIB, called SI-SAP-QID MIB based on the BSM SI-SAP performance parameters defined in [2]. $\mathrm{D} / \mathrm{B}_{1}$ may also contain other data structures, e.g. technology or vendor specific ones.

$\mathrm{D} / \mathrm{B}_{2}$ contains a newly specified BSM-specific MIB, called IPperf MIB based on the BSM IP performance parameters defined in [2]. $\mathrm{D} / \mathrm{B}_{2}$ may also contain other data structures, at the wish of the BSM network operator. $\mathrm{D} / \mathrm{B}_{2}$ is in fact the interface between the BSM-internal and external worlds; it will most likely be a combination of data elements or data structures (some standard ones, some proprietary ones, and some BSM-specific ones).

Visibility (read/write access rights) of the databases should be regulated by the $\mathrm{BSM}$ network operator; e.g. $\mathrm{D} / \mathrm{B}_{2}$ may or may not be made visible to external parties. In any case it should be noted that for specific data elements it may not be possible to prevent external parties from directly polling $\mathrm{D} / \mathrm{B}_{1}$.

There is therefore a minimum set of element management data which are to be standardized and should be provided by all BSM compliant systems. They could thus be accessed by means of standard SNMP through the NMC (see Figure 2). So service and network performance considerations can be derived by aggregation and/or other types of analysis.

The BSM network should also foresee a central PM server which accomplishes its tasks interacting with PM modules (or PM traffic nodes) located at the STs. The server is responsible for selecting measurements to be performed, configuring the PM nodes to perform them, for collecting the data, and for the final performance assessment. The PM server may or may not be involved in the measurement as it is shown in Figure 2. This centralized architecture, where a central server is responsible for the PM in the network, seems quite suitable to a satellite network.

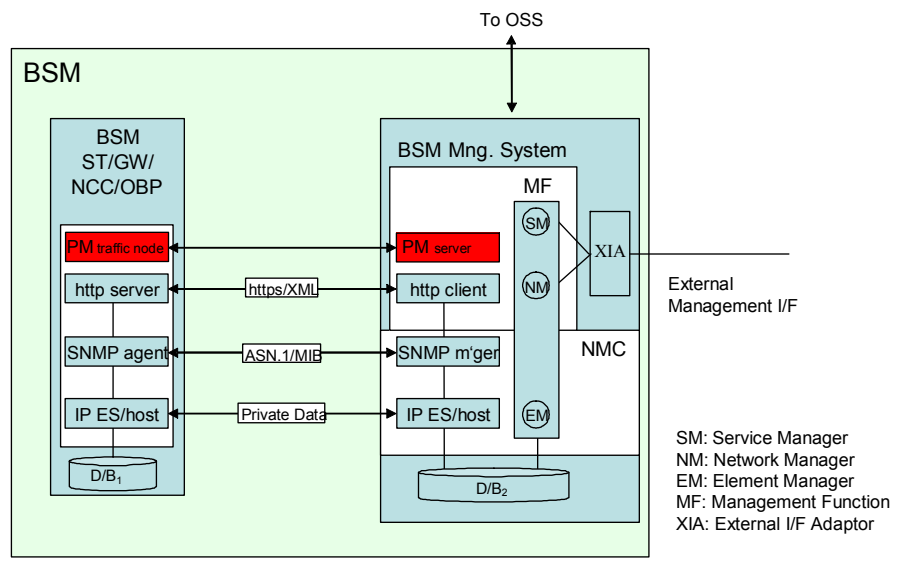

Figure 2: BSM Management Functional Architecture

\section{HIERARCHY (ACTORS AND ROLES)}

\section{A. Performance Management Operations}

In a centralized architecture, as the one presented in the previous chapter, the following four PM operations can be clearly identified:

- $\quad$ selection of the measurements to be performed,

- configuration of the PM nodes which perform them,

- measurements execution and collection of the data,

- $\quad$ final performance assessment.

They will be detailed in the next clause.

\section{B. Roles}

The four operations listed above include a series of subtasks which should be performed by different entities in the network (either by the PM server, or by the PM traffic nodes, or by both). In this clause we distribute these tasks to the different PM roles in the network and describe the details.

\section{1) PM traffic node (ST)}

The following activities may be performed by the PM traffic nod (ST) in the four operation areas identified above:

- Selection of the measurements to be performed: the ST should decide to perform selected measurements independently only if this is required to populate local databases, or in order to fulfill requirements given by the central PM server (e.g. keep a given parameter upto-date).

- configuration of the PM nodes which perform measurements: the PM traffic node may be preconfigured with test applications, or may be completely configurable from remote (e.g. by RMON), both these options are possible either for active and for passive measurements.

- measurements execution and collection of the data: Normally the ST will populate the database D/B1 with the results of the performed measurements. The ST is involved in the measurement of parameters when (or collection of measurement data):

- it is the only MP for the parameter, so the parameter is measured locally (e.g. number of active QIDs, see [2]);

- $\quad$ it is involved in a measurement which takes place between multiple MPs, typically one-way measurements (e.g. one-way delay by means of OWAMP);

- $\quad$ it is exporting data, which are collected by some other entities in the network, if, for example, IPFIX is used, the ST will run an IPFIX exporter.

- final performance assessment: not relevant for a PM traffic node. 


\section{2) PM server (BNMS)}

The following activities may be performed by the PM traffic nod (ST) in the four operation areas identified above:

- Selection of the measurements to be performed: the PM server selects the metrics to be measured, i.e. the singleton metrics (in IETF ippm terminology); it also decides the domain of measurements and the way of sampling metrics:

- E.g. which STs? Which applications? Which network sections? Observation time, sampling period, etc.,

- Decides how to derive the sample metrics (in IETF ippm terminology).

- configuration of the PM nodes which perform measurements: the PM server takes care of configuring the PM traffic nodes to instruct them on how to measure parameters, either for passive monitoring or for active measurements; if a pre-processing has to be done at the ST (i.e. on some sample metrics to derive statistical metrics, in IETF ippm terminology) this is also configured by the PM server.

- measurements execution and collection of the data: Normally the PM server will populate the database $\mathrm{D} / \mathrm{B} 2$ with the results of measurements. If it is required to process the collected data for some statistics before writing them in the database, the PM is also responsible for that. It is also involved in the measurement of parameters when (or collection of measurement data):

- $\quad$ it is involved in a measurement which takes place between multiple MPs, typically one-way measurements (e.g. one-way delay by means of OWAMP); it may be frequent to involve the PM server in one-way measurements;

- it is collecting data, which are exported by some other entities in the network (i.e. the PM traffic nodes), if, for example, IPFIX is used, the PM Server will run an IPFIX collector (see Figure 3).

- final performance assessment: The PM performs all the relevant analysis on the collected data to derive statistics and/or general consideration on the overall network performance; if needed, it can also perform some kind of pre-processing before writing the outcome of this analysis onto the database.

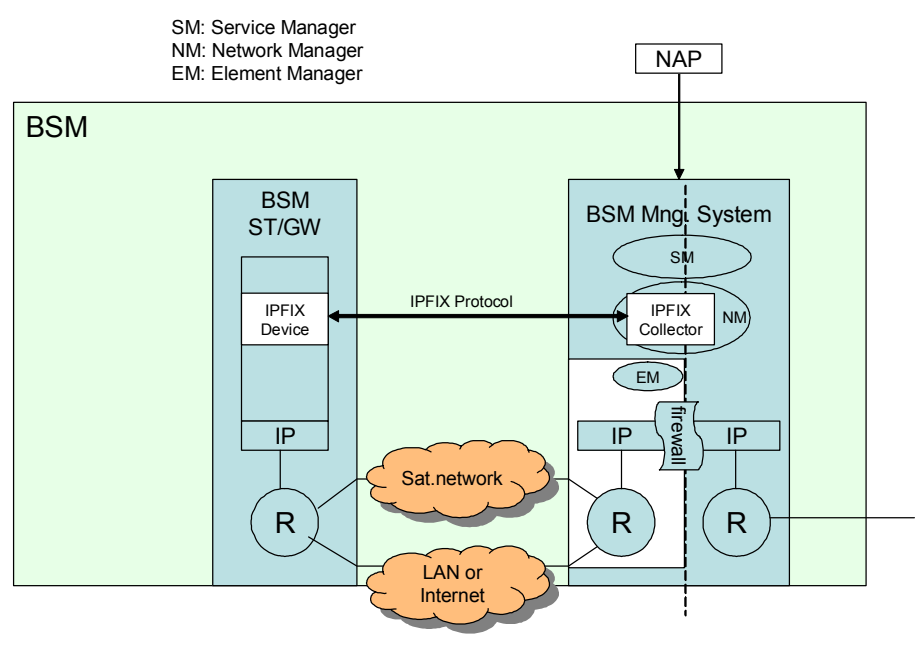

Figure 3: BSM PM Server Architecture (IPFIX example)

\section{CONCLUSION}

The strategy for Performance Management in satellite networks has been outlined. The ETSI BSM WG is using these ideas to generate a standard for Performance Management with industry feedback.

\section{REFERENCES}

[1] ETSI TS 102 672: "Satellite Earth Stations and Systems (SES); Broadband Satellite Multimedia (BSM): Management Functional Architecture".

[2] ETSI TR 102 673: "Satellite Equipment and Systems (SES); Broadband Satellite Multimedia; Performance Parameters".

[3] ETSI TS 102 462: "Satellite Earth Stations and Systems (SES); Broadband Satellite Multimedia (BSM); QoS Functional Architecture".

[4] ETSI TS 102 463: "Satellite Earth Stations and Systems (SES); Broadband Satellite Multimedia (BSM); Interworking with IntServ QoS".

[5] ETSI TS 102 464: "Satellite Earth Stations and Systems (SES); Broadband Satellite Multimedia (BSM); Interworking with DiffServ QoS".

[6] ITU-T M.2301, "Performance objectives and procedures for provisioning and maintenance of IP-based networks".

[7] RFC 2330: "Framework for IP Performance Metrics".

[8] RFC 2678: "IPPM Metrics for Measuring Connectivity".

[9] RFC 2679: "A One-way Delay Metric for IPPM".

[10] RFC 2680: "A One-way Packet Loss Metric for IPPM".

[11] RFC 2681: "A Round-trip Delay Metric for IPPM".

[12] RFC 3418: "Management Information Base (MIB) for the Simple Network Management Protocol (SNMP)". 